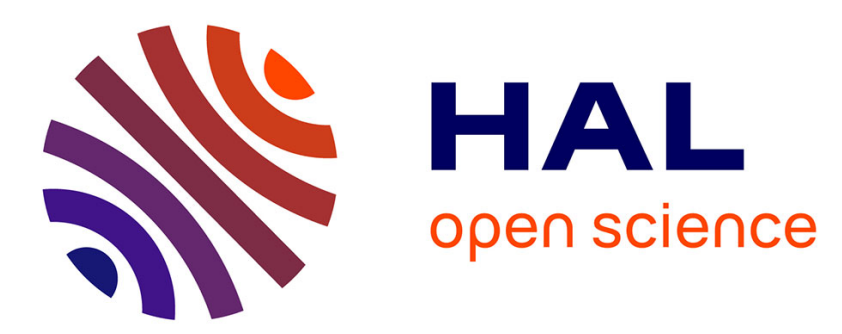

\title{
Ab initio Methods for Surfaces and Interfaces: Status and Perspectives
}

E. Wimmer

\section{To cite this version:}

E. Wimmer. Ab initio Methods for Surfaces and Interfaces: Status and Perspectives. Journal de Physique IV Proceedings, 1997, 07 (C6), pp.C6-75-C6-88. 10.1051/jp4:1997607 • jpa-00255706

\section{HAL Id: jpa-00255706 https://hal.science/jpa-00255706}

Submitted on 1 Jan 1997

HAL is a multi-disciplinary open access archive for the deposit and dissemination of scientific research documents, whether they are published or not. The documents may come from teaching and research institutions in France or abroad, or from public or private research centers.
L'archive ouverte pluridisciplinaire HAL, est destinée au dépôt et à la diffusion de documents scientifiques de niveau recherche, publiés ou non, émanant des établissements d'enseignement et de recherche français ou étrangers, des laboratoires publics ou privés. 


\title{
$A b$ initio Methods for Surfaces and Interfaces: Status and Perspectives
}

\author{
E. Wimmer \\ Molecular Simulations, SARL, Parc Club Orsay Université, 20 rue Jean Rostand, 91893 Orsay, France
}

\begin{abstract}
Many structural, physical, and chemical properties of surfaces and interfaces can now be described by computational approaches at a remarkable level of accuracy and detail, thus helping in the interpretation of experimental data and in the search for novel systems. This contribution provides an overvicw of the major theoretical and computational methods with an emphasis on ab initio density functional methods and their implementations. The current capabilities are illustrated by the following examples: the reactive adsorption of $\mathrm{SiH}_{4}$ on a $\mathrm{Si}(001)$ surface, the surface reconstruction of the $\beta$-SiC $(001)$ surface, and magnetism on transition metal surfaces and interfaces. A discussion of the current obstacles and a general outlook on the evolution of ab initio methods conclude this contribution.
\end{abstract}

\section{INTRODUCTION}

We are presently witnessing a Golden Age for atomistic studies of surfaces and interfaces. There are three major factors which have created this exciting situation, namely (i) the increased technological importance of surfaces and interfaces, (ii) the development of sophisticated experimental surface techniques, and (iii) the availability of theoretical and computational tools which allow an understanding of surface phenomena at an unprecedented level of detail and accuracy. It is the aim of the present contribution to put these computational approaches in perspective, to discuss their underlying concepts, and to highlight the present capabilities.

A major factor for the technological interest in surface and interface phenomena comes from microelectronics and related areas such as recording and display technologies where a detailed understanding and control of surfaces and interfaces on the atomic level is a prerequisite for the continuing successful miniaturization of components and devices. In addition, many other areas in materials science and chemistry such as catalysis, adhesion, tribology, corrosion, and composite materials have made the understanding of surfaces and interfaces the focus of intense research and development efforts.

Experimentally, ultra-high vacuum techniques have made it possible to prepare surfaces with extremely high purity and low levels of defects. The development of novel surface techniques, notably those exploiting synchrotron radiation and real-space probes such as scanning tunneling microscopy (STM) have lead to a wealth of new experimental data. New generations of synchrotrons have started their operation or are under construction producing high-resolution data at unprecedented rates thus opening the possibility for the study of time-dependent surface phenomena.

At the same time, the development of powerful theoretical methods and efficient algorithmic implementations has been fueled by the tremendous evolution in speed, capacity, ease of use, and the decreasing cost of computer hardware. Together, these developments have created a unique and most exciting situation for the computational study of surfaces and interfaces.

Besides their practical importance outlined above, surfaces and interfaces offer a range of fundamental scientific aspects such as the occurrence of surface reconstructions, surface phase transitions, electronic surface and interface states, and unexpected magnetic behavior. In addition, the study of surface and interface processes sets the stage for linking biological systems to the domains of synthetic materials and microelectronic devices. The detailed investigation of surface and interface phenomena is crucial for a deeper understanding of the organizing principles which govern the link between atomistic processes, mesoscopic structures, and macroscopic properties. There is no doubt that computational approaches will play an increasingly important role in pursuing these efforts. 
Any theoretical/computational approach to surfaces and interfaces encounters two fundamental challenges as shown in Fig. 1, namely the gaps in the length scale and the time scale which separate the atomistic domain from the macroscopic world. The challenge of the length scale is obvious, but perhaps the gap in the time scale is actually more difficult to bridge. The characteristic time of atomistic processes such as vibrational motions of atoms or electronic transitions take place in the order of femto seconds whereas macroscopic processes on surfaces such as the growth of semiconductors by chemical vapor deposition or a catalytic process is measured in the time scale of seconds. Other surface processes such as corrosion may in fact involve time scales which are many orders of magnitude longer.

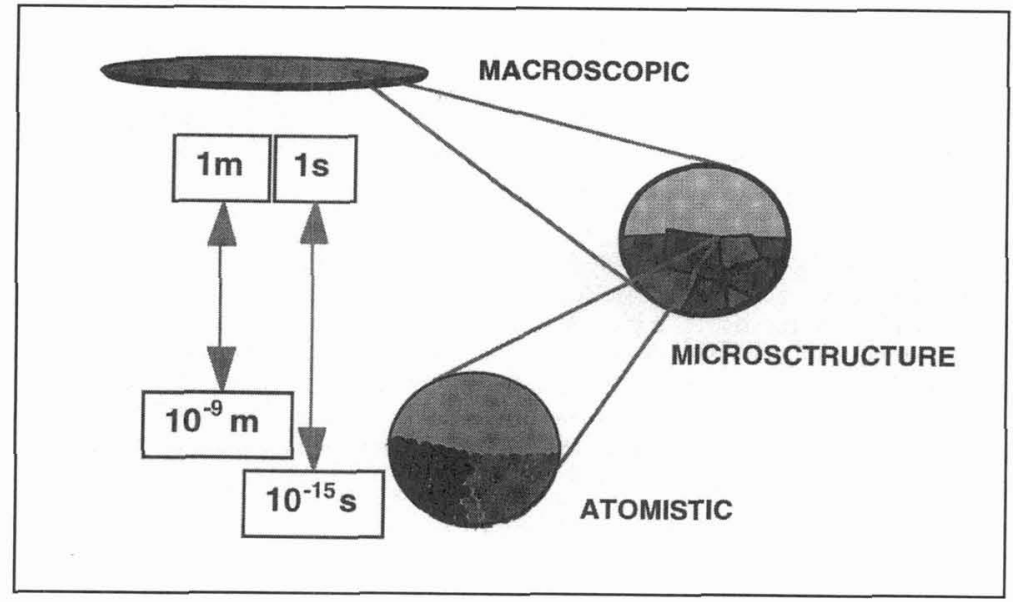

Figure 1: Characteristic macroscopic versus atomistic length and time scales. Note that the gap in the time scale is significantly larger than that in the length scale.

\section{THEORETICAL AND COMPUTATIONAL CONCEPTS}

The central issue of any atomistic simulation of surfaces and interfaces is the determination of the equilibrium positions of the atoms and their trajectories associated with dynamic processes. To this end, one needs to know the total energy, $E$, as a function of any possible arrangement of the atoms.

$$
E=E\left[\mathbf{R}_{1}, \mathbf{R}_{2}, \ldots \mathbf{R}_{\mathrm{n}}\right]
$$

Here, $\mathbf{R}_{1}, \mathbf{R}_{2}, \ldots \mathbf{R}_{\mathrm{n}}$ denote the Cartesian coordinates of all atoms in the system under consideration. In most cases, one can separate the motions of the electrons from those of the nuclei. In this well-known BornOppenheimer approximation, only the electrons are treated quantum mechanically whereas the atomic nuclei are described classically by Newton's equations of motion. The trajectory of each atom, $\alpha$, is therefore determined by

$$
\mathbf{a}_{\alpha}=\frac{1}{m_{\alpha}} \mathbf{F}_{\alpha}=-\frac{1}{m_{\alpha}} \nabla_{\alpha} E
$$

where $\mathbf{a}_{\alpha}$ is the acceleration of atom $\alpha$ of mass $m_{\alpha} ; \mathbf{F}_{\alpha}$ is the force acting on this atom in the direction of the gradient of the potential energy surface, $E$.

The evaluation of the potential energy surface consists in the solution of the many-electron problem using a quantum mechanical approach. Alternatively, one can also neglect the explicit treatment of electrons and replace their action by interatomic potentials or force fields. Depending on the nature of the system, the type of physical or chemical property of interest, and the desired accuracy, a range of methods have been developed to describe the interatomic interactions.

Given a description of the interatomic interactions as outlined above, the steps and choices involved in a typical computational approach to a problem in surface or interface science are illustrated in Fig. 2. One of the most critical steps is the proper choice of the geometric model. Conceptually, a semi-infinite crystal is 
the most satisfying representation of a surface. By using Green's function embedding and matching techniques, the electronic structure can be solved for this geometry. However, the computational effort of such an approach is rather large. A single slab geometry is another viable geometric choice, provided that the slab is sufficiently thick to achieve bulk-like behavior in the interior without spurious interactions between the two surfaces of the slab. For transition metals, at least five layers are necessary to obtain a reasonable description. For $s, p$-metals such as aluminum, more than ten layers are required to suppress the artificial interactions between the two surfaces of the slab. A similar number of layers is necessary to describe semiconductors. Single slab geometries have been implemented in programs such as the linearized augmented plane wave method. With the development of iterative schemes and fast-Fourier techniques using pseudopotential plane wave methods, it is computationally very convenient to retain threedimensional periodicity. Therefore, a super-slab geometry has become very common. In this model, slabs consisting of five to about twenty atomic layers are stacked in the direction perpendicular to the surfaces leaving a spacing corresponding to about three to five atomic layers between each slab thereby creating a "vacuum" region. This model is appropriate for bare, flat surfaces, but becomes cumbersome when one describes, for example, the adsorption of large molecules on a surface.

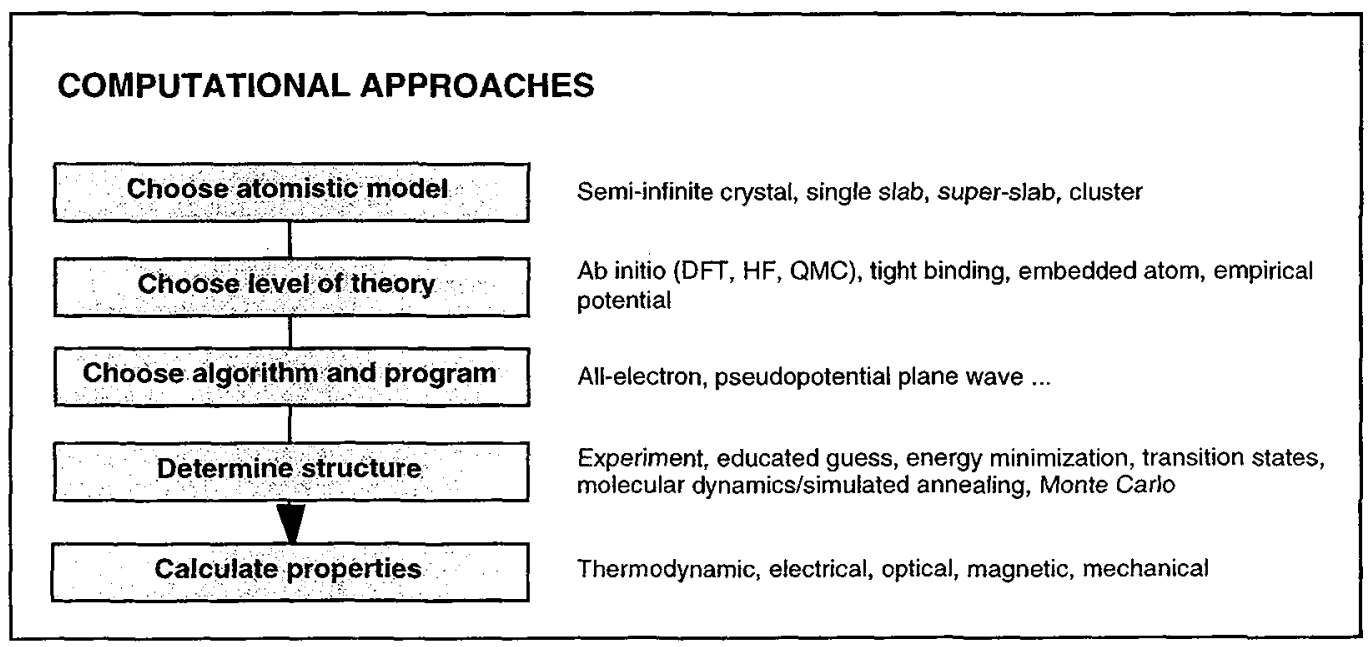

Figure 2: Steps and choices in a typical computational study of surface or interface phenomena. Possible levels of theory include density functional theory (DFT), Hartree-Fock (HF) and quantum Monte Carlo (QMC) as explained in the text.

A cluster model of surfaces and interfaces allows the use of standard molecular methods. However, the termination of the cluster is a critical aspect and one needs to be very careful that boundary effects do not cloud the actual surface effects under investigation. In the case of covalent systems such as silicon or diamond, one can terminate the bonds towards the bulk with hydrogen atoms. In the case of ionic crystals such as $\mathrm{MgO}$, it is mandatory to include the long-range Coulombic interactions. This can be accomplished by adding the external field of point charges to the quantum mechanical Hamiltonian. However, the effect on the electronic wave functions on the boundary of the cluster needs to be carefully controlled. For metallic systems with itinerant wave functions, quite large clusters containing hundreds of atoms would be necessary to approach the situation on a semi-infinite surface. At present this would lead to unacceptable computational requirements in terms of computer time and memory.

In selecting the appropriate level of theory, one needs to balance the desired level of accuracy and detail with the computational effort and the size of the model. The present contribution focuses on quantum mechanical ab initio methods based on density functional theory, since this approach offers a good balance between accuracy, computational effort, and general applicability. Organic molecules and ionic systems such as $\mathrm{MgO}$ are well described by Hartree-Fock theory. Whereas a large number of quantum chemical programs exist for the study of isolated organic molecules, Hartree-Fock methods for solids and surfaces play a minor role compared with density functional calculations. Quantum Monte Carlo (QMC) methods are gaining in importance as an alternative to high-level correlated ab initio methods such as coupled cluster approaches. However, these accurate quantum mechanical methods can currently be used only for systems 
containing about ten to twenty atom and are therefore not directly applicable to surface problems. On the other hand, simplified quantum mechanical methods such as tight binding theory, embedded atom methods, or empirical potentials may currently offer the only practical framework for problems which require a large number of atoms or the investigation of dynamical processes such as diffusion and growth. For a further discussion of the present theoretical and computational methods the reader is referred to recent articles $[1,2]$ and references therein.

\subsection{Density Functional Theory}

Within the scope of this contribution, only the most relevant aspects of density functional theory (DFT) are highlighted. For an in-depth treatment of density functional theory the reader is referred to the literature, for example reference [3].

DFT provides a rigorous framework for the quantum mechanical description of any ensemble of atoms such as surfaces and interfaces containing any atom from the periodic table. Perhaps the most important practical problem that can be solved with DFT is the determination of the changes in the total energy of a system as a function of the positions of the atoms. As stated above, this knowledge of the energy hypersurface is fundamental to the understanding of any surface system. It allows the prediction of surface reconstructions, the prediction of vibrational spectra, the determination of adsorption geometries of molecules and, at least in principle, the description of transition state geometries and barrier heights of any surface reaction.

In DFT [4,5] the total energy is expressed as a functional of the electron density, $\rho$, for given positions, $\mathbf{R}_{c}$ of all atomic nuclei in the model.

$$
E=E\left[\rho(\mathbf{r}), \mathbf{R}_{\alpha}\right]
$$

This functional has a minimum for the ground state electron density.

$$
\left.\frac{\partial E[\rho]}{\partial \rho}\right|_{\rho=\rho_{0}}=0
$$

The total energy is decomposed into three contributions, a kinetic energy, $T_{o}$, a Coulomb energy, $U$, due to classical electrostatic interactions among all charged particles in the system, and a term called the exchange-correlation energy, $E_{x c}$, that captures all many-body interactions.

$$
E=T_{o}+U+E_{x c}
$$

This decomposition is formally exact, but only the relationship between the Coulomb energy and the total charge distribution can be given directly. For any practical purposes, the calculation of the kinetic energy term requires good one-particle wave functions and the exchange-correlation term needs to be approximated.

In wave function based quantum chemical methods such as Hartree-Fock theory with second order perturbation theory, coupled-cluster methods and configuration interaction (CI) methods, the high and often prohibitive computational effort comes from the specific description of electron correlation. In fact, these methods implicitly assume that these many-body effects of the interacting electron system are of a longrange delocalized nature.

The local density approximation (LDA) of density functional theory and also the more recent generalized gradient approximations (GGA) rest on the assumption that electrons are essentially "nearsighted" [6]. It turns out that this assumption is justified especially in regions of reasonably high electron density as found, for example, in a solid and a surface. Therefore, as an approximation, the exchange-correlation energy is taken from known results of an interacting electron system of constant density ("homogeneous electron gas") and it is assumed that the exchange and correlation effects are not strongly dependent on inhomogeneities of the electron density in the vicinity of the reference point, $\mathbf{r}$. Thus, the local electron density can be used to evaluated the exchange and correlation effects of a volume element around $\mathbf{r}$ by the following expression

$$
E_{x c}^{L D A}[\rho] \approx \int \rho(\mathbf{r}) \varepsilon_{x c}^{o}[\rho(\mathbf{r})] d \mathbf{r}
$$


The exchange-correlation energy per electron, $\varepsilon_{\mathrm{xc}}^{\prime \prime}$, in a system of interacting electrons of constant density is well known [7]. It turns out that the LDA is a surprisingly good approximation and many structural properties and relative energy changes of a great variety of systems are very well described. However, it has been found for many systems that atomization energies are overestimated by the LDA. Thus, the calculation of absolute values for binding energies, but also dissociation energies and weak interactions such as hydrogen bonds or carbonyl bonds require methods beyond the local density approximation. One possibility is offered by gradient corrections such as those suggested by Becke $[8,9]$, Perdew [10] and Lee et al. [11]. In these approaches, terms depending on the gradient of the electron density are included in the expressions for the exchange and correlation energy.

$$
E_{x c}^{G G A}=f[\rho,|\nabla \rho|]
$$

Therefore this level of theory is often referred to as generalized gradient approximation (GGA). While the GGA improves the absolute values of binding energies of most compounds as well as the bond distances in many weakly bound systems, there is no guarantee that the GGA results, especially geometries but also relative energies, are always systematically better than those obtained at the LDA level.

The accuracy of DFT calculations is intimately related to the use of one-particle wave functions, $\psi_{i}$, which define the total electron density through

$$
\rho(\mathbf{r})=\sum_{i} n_{i}\left|\psi_{i}(\mathbf{r})\right|^{2}
$$

and the kinetic energy of the electrons by

$$
T_{o}=\sum_{i} n_{i} \int \psi_{i}^{*}(\mathbf{r})\left(-\frac{1}{2} \nabla^{2}\right) \psi_{i}(\mathbf{r}) d \mathbf{r}
$$

with $n_{i}$, being the occupation of level $i$. Using this decomposition of the total electron density into oneparticle densities, the variational properties given by eqs. (3) and (4) lead to effective one-particle Schrödinger equations, which are referred to as Kohn-Sham equations

$$
\left[-\frac{1}{2} \nabla^{2}+V_{C}(\mathbf{r})+\mu_{x c}(\mathbf{r})\right] \psi_{i}(\mathbf{r})=\varepsilon_{i} \psi_{i}(\mathbf{r})
$$

Corresponding to the three terms in the total energy expression (5), namely the kinetic energy, the Coulomb energy, and the exchange-correlation energy, the effective one-particle Hamiltonian of the Kohn-Sham equations contains a kinetic energy operator, a Coulomb potential operator, and an exchange-correlation operator. The latter is related to the exchange-correlation energy by

$$
\mu_{x c}=\frac{\partial E_{x c}[\rho]}{\partial \rho}
$$

The theory has been generalized to spin-polarized systems $[12,13]$ in which the density of the spin-up electrons and that of the spin-down electrons are different, as is the case in magnetic systems. In practice this means that the Kohn-Sham equations have to be solved for the spin-up and spin-down electrons individually.

The fundamental quantities of DFT are the total electron density (and spin density for spin-polarized systems) and the corresponding total energy. The one-particle wave functions (i.e. molecular orbitals in the case of molecules) and the associated one-particle eigenvalues are, strictly speaking, only auxiliary quantities. In practice, however, these one-particle quantities are extremely useful in order to explain surface states, to distinguish between metallic and insulating behavior of a solid, and to interpret optical excitation energies and photoemission experiments or even subtle effects such as the magneto-optic Kerr effect and magnetic anisotropy energies. For metallic and delocalized systems, the eigenvalues actually correspond almost quantitatively to excitation energies. In fact, the highest occupied level can be interpreted directly as the negative of the ionization energy or work function. In semiconductors, where localization can be important, the LDA gap is typically $30-50 \%$ too small compared with the experimental optical gap. For very localized systems such as $f$-electrons in rare-earth and actinide compounds, these one-particle 
eigenvalues are far from excitation energies. In order to describe these localization and correlation effects to predict excitation phenomena, one has to go beyond the LDA. The possibilities include the GW method [14], the so-called LDA+U methods [15], self-interaction corrections (SIC) [16], optimized effective potentials (OEP) and time-dependent density functional theory [17].

In the search for minimum energy configurations such as surface reconstructions or adsorption sites of molecules on a surface as well as for molecular dynamics studies, it is necessary to know not only the total energy for a given arrangement of the atoms, but also to have values for the force vectors acting on each atom. Formally, this quantity is derived by differentiating the expression (5) with respect to the displacement of a nucleus. The actual evaluation is straightforward within a pseudopotential plane wave approach, but can be algorithmically quite involved for localized basis sets or linearized augmented plane wave approaches. In the past five to eight years, this feature has become a standard feature of many DFT implementations.

If a system contains atoms with an atomic number larger than about 50 , relativistic effects are important and should be included. Density functional theory has been generalized and successfully applied to relativistic systems, either in the form of pseudopotentials which incorporate relativistic effects, or in the form of semi-relativistic and fully relativistic implementations [18].

\subsection{Implementations of DFT}

At present, there is not a single DFT implementation which would be equally applicable and efficient for all systems ranging from molecules to magnetic transition metal heterostructures. Therefore, a number of different approaches have emerged as shown in Fig. 3. Following the scheme shown in Fig. 3, the key features of the most common approaches for the $a b$ initio calculation of surface structures are discussed below.

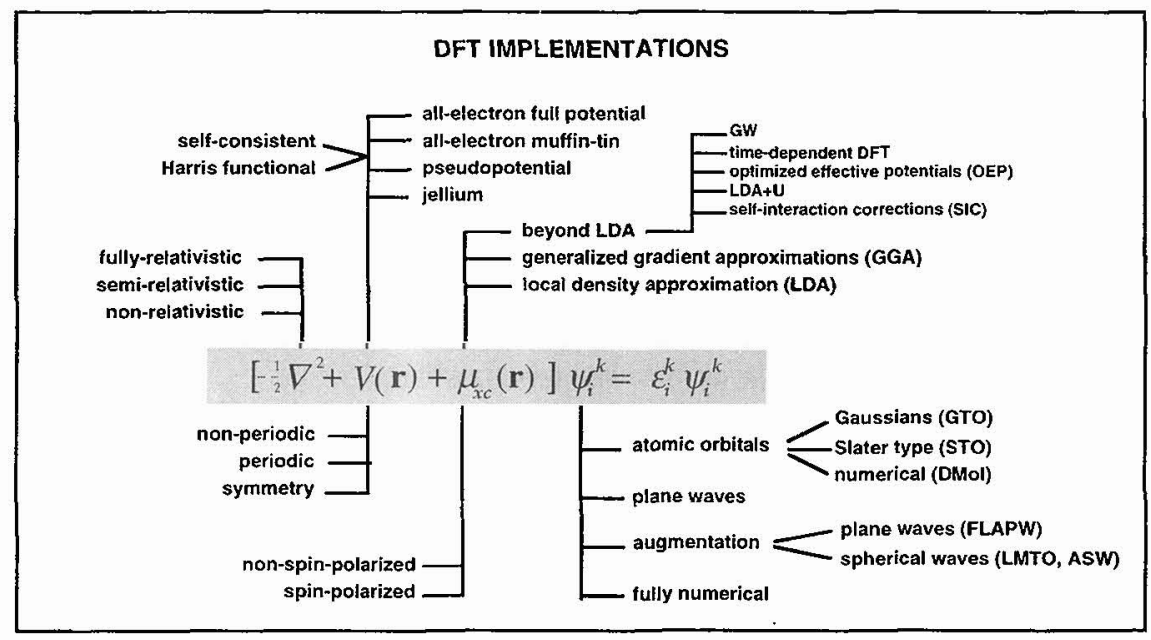

Figure 3: Implementations of density functional theory for the calculation of the crystallographic and electronic structures of surfaces and interfaces. Particular methods are usually named after the choice of the form of the potential and the basis set such as the pseudopotential plane wave method and the full-potential linearized augmented plane wave (FLAPW) method.

\subsubsection{Pseudopotential Plane Wave Method}

The concept of pseudopotentials has been used since the early days of band structure calculations. The major convenience of this approach is the ability to use plane wave basis sets, which are mathematically elegant and their implementations in computer programs are relatively simple. For a fairly long time, pseudopotentials were only applied to atoms such as $\mathrm{Si}, \mathrm{Ge}, \mathrm{Ga}$, and As for which smooth pseudopotentials can be defined. A serious disadvantage of plane waves is their slow convergence. If one uses a matrix diagonalization procedure to find the eigenvalues, this slow convergence limits the 
application of plane wave methods to fairly small unit cells containing of the order of ten atoms. With the introduction of ab initio molecular dynamics by Car and Parrinello [19], a concept which unified electronic structure calculations with molecular dynamics and, at the same time, circumvented the direct matrix diagonalizations. Pre-conditioned conjugent gradient methods have been demonstrated as another way to avoid these diagonalizations as reviewed by Payne et al. [20]. This opened the possibility to use significantly larger basis sets. Furthermore, pseudopotential plane wave methods were extended to transition metals. However, the treatment of rare-earth elements and actinides with their localized $f$ electrons remains the domain of all-electron methods.

Today, pseudopotential plane wave methods have become a work-horse for the study of structural and electronic properties of surfaces and interfaces. Typically, unit cells in the super-slab geometry containing 50-100 atoms can be treated for geometry optimizations. Larger systems containing several hundreds of atoms are feasible, but computer memory requirements and computer time become serious limitations. Examples of recent applications to surface problems include the site-selective adsorption of $\mathrm{C}$ atoms on $\mathrm{Al}(111)$ surfaces [21] and the study of $\mathrm{O}$ ad-layers on $\mathrm{Ru}(0001)$ [22].

Pseudopotential plane wave methods have found particularly widespread use in the study of semiconductors such as $\mathrm{Si}, \mathrm{Ge}$, and GaAs. One of the reasons is the fact that very efficient and accurate pseudopotentials can be constructed for these main group elements. On the other hand, pseudopotential plane wave approaches for the systems with transition metals have only recently been investigated and the treatment of rare earth and actinides is questionable. In fact, the localized nature of $d$-states and especially $f$-electrons suggests the use of localized basis functions. On the other hand, one would like to keep the advantages of plane wave basis sets. Such considerations have lead to the concept of augmented plane waves, which is discussed next.

\subsubsection{Full-potential Linearized Augmented Plane Wave (FLAPW) Method}

In 1937 Slater [23] introduced the concept of augmented plane waves (APW) in electronic structure calculations. The basic idea consists of dividing the real space in spherical regions around the atoms and an interstitial region. The "natural" form of the wave function is used in each region. Matching conditions on the boundaries between the "muffin-tin" spheres and the interstitial region ensure the continuity of the wave functions. This concept has turned out to be extremely powerful and even today, the descendants of the original APW method belong to the most accuracy approaches for surface electronic structure calculations. Major steps in the development of this method were the linearization of the eigenvalue problem [24,25], the extension to surface problems [26], the incorporation of the full potential [27], the development of total energy capabilities [28], and the more recent efforts to calculate forces $[29,30]$. The FLAPW method has been implemented in several programs and is being further developed, e.g. [31] thus demonstrating the viability and interest in this method.

The current implementations of the full-potential linearized augmented plane wave method use a scalarrelativistic Hamiltonian with the option to include spin-orbit relativistic effects in the form of a second variation. Calculations for surfaces and interfaces are performed either in a single-slab geometry or in the form of a three-dimensional super-slab. The calculation of magnetic properties is a particular strength of this method [32,33] and therefore it contains spin-polarized as well as non spin-polarized options within the LDA or GGA treatment for exchange and correlation.

\subsubsection{Linearized Muffin-Tin Orbital (LMTO) and Augmented Spherical Wave (ASW) Methods}

The essence of the LAPW method is the use of atomic-like functions around each atomic center. These functions are truncated at the sphere boundaries. Plane waves in the interstitial region link these functions by imposing the continuity conditions. In this way, information from one atomic center to all others is transmitted via plane waves. This approach eliminates the problem of tails overlapping with neighboring atoms which can cause a computational bottleneck for closed-packed solids if one would use just atomiclike functions. Based on the concepts of the Korringa-Kohn-Rostoker (KKR) method [34,35], one can use scattering functions to describe the wave functions in the interstitial region. In its linearized form, this approach is know as the linearized-muffin-tin-orbital (LMTO) method [36]. Related to this method is the augmented-spherical-wave (ASW) approach [37].

These approaches are particularly well suited for the study of the electronic structure of close-packed solids containing transition metals and rare earths elements. These methods are fast, but in general not suited for geometry optimizations. This is related to the atomic-sphere approximations (ASA) used in typical LMTO or ASW calculations. The idea of the ASA is as follows. The atomic spheres around each 
atom are chosen such that the sum of the volumina of all spheres equals the total volume of the unit cell. Obviously, this leads to overlapping spheres. Experience has shown that this approximation is justifiable as long as the overlap region is not more than about $15 \%$ of the unit cell volume. In each of the spheres, only the spherically symmetric part of the effective potential is used for the calculation of the energy band structures and densities of states. Because of this simplification, LMTO and ASW calculations are about one order of magnitude faster compared with FLAPW calculations. However, their use for surface calculations is limited whereas this approach is well suited for interfaces between closed-packed metals. The LMTO and ASW methods can be generalized to full-potential methods $[38,39]$ at the cost of a substantial increase in computational effort.

\subsubsection{Linear Combination of Atomic Orbital (LCAO) Methods}

In nature there is no sharp boundary between molecules, small particles, and extended solids with their surfaces. Therefore, electronic structure methods which are successful in describing molecules should also be applicable to solids and surfaces. The vast majority of molecular electronic structure calculations are based on linear combinations of atomic orbitals. Three types of functions have emerged as preferred choices to represent the atomic orbitals, namely Gaussian-type functions, Slater type orbitals, and numerical functions.

Gaussian basis sets. Since their introduction in the early 1950 's, Gaussian basis sets have become the standard in quantum chemistry. To a large extent, this choice was dictated by the occurrence of difficult four-center integrals in Hartree-Fock calculations, which require a mathematically and computationally efficient solution. Gaussian functions meet these requirements. For similar reasons, CRYSTAL [40] which is a Hartree-Fock program for periodic solids, is also based on Gaussians.

In density functional calculations, Gaussians have been successfully implemented for electronic structure calculations of solids and surfaces $[41,42]$. For molecular density functional calculations, the use of Gaussian-type orbitals has been pioneered by Sambe and Felton [43] and by Dunlap [44]. Further developments have lead to programs such as DeMon [45] and DGauss [46].

The use of Gaussians for surface calculations is fairly efficient in the case of covalent systems such as semiconductor surfaces, as demonstrated, for example, in the recent studies on the reconstruction of the $\mathrm{SiC}(001)$ surface [47]. Gaussians do not have the correct behavior of wave functions in the limits close to the nuclei and at large distances. The nodal structure of the radial part of the electronic wave functions has to be introduced by a superposition of several Gaussians with appropriate exponents. At large distances, Gaussians decay too quickly. Pseudopotentials as used, for example in [47] alleviate some of the problems. However, the unphysical character of Gaussians requires great care in the choice of the basis sets. This problem has been extensively discussed in the context of Hartree-Fock calculations [48] and it persists in density functional methods [49].

Slater-type orbitals. While Hartree-Fock methods are practically limited to Gaussian-type orbitals, a variety of other basis functions can be employed for density functional calculations. One of the options are Slatertype orbitals (STO's). These functions are analytically defined as are Gaussians, but the shape of STO's is closer to actual atomic orbitals. The use of STO's in molecular density functional calculations was developed in the 1970's [50,51] and has led to programs such as the Amsterdam Density Functional (ADF) code applicable to systems with 1D, 2D, and 3D periodic boundary conditions [52].

Compared with a free atom, the one-particle wave functions in a condensed phase are more compressed. The degree of the radial compression depends on the particular chemical environment. In order to provide the variational freedom to accommodate such a compression, one takes at least two radial functions for the representation of each atomic orbital such as an O-2p function. In the case of STO's this requires two different values of $\zeta$ per orbital with quantum numbers $n \ell$ Hence this choice is called a double-zeta basis. In fact, this terminology is also being used in conjunction with Gaussian-type orbitals, since Gaussians can be used to mimic Slater-type orbitals. The concept of two radial functions for each $n \ell$ is quite general and occurs in a variety of methods including the FLAPW method.

Despite the analytical form of STO's, current density functional programs such as the ADF code evaluate the Hamiltonian and overlap matrix elements by a numerical integfation. This is in contrast to Gaussian-based methods, where all matrix elements except those of the density functional exchangecorrelation potential operator are usually evaluated analytically.

Numerical atomic orbitals. In most density functional implementations the exchange-correlation terms are evaluated on a numerical grid. Therefore, it seems quite appealing to use numerical representations and 
numerical integrations also for all other terms. In particular, one can employ numerically defined atomic orbitals instead of analytical forms such as Gaussians and STO's. This approach has been pioneered by Averill and Ellis [53] and Delley and Ellis [54]. Subsequent developments by Delley [55,56] have lead to the DMol program and its generalization to periodic systems (DSolid).

Numerical atomic orbitals are generated by solving the Kohn-Sham equations for isolated atoms. This procedure yields functions which have automatically the correct nodal behavior close to the nucleus and an exponential decay at larger distances. In fact, to any desired degree of numerical accuracy these functions are the "exact" solutions to the atomic Kohn-Sham Hamiltonian.

As stated above, the wave functions in a condensed system tend to be more compressed than those of an isolated atom. To this end, one uses for each orbital with quantum numbers $n \ell$ at least two radial numerical functions, one corresponding to the isolated neutral atom and the other obtained from a positive ion. For example, to describe a Si-3s function, on takes the $3 s$ orbital from a neutral Si atom and a second $3 s$ function from a $\mathrm{Si}^{2+}$ ion. The function from the positive ion is more compressed than the function from the neutral atom, thus providing the necessary variational freedom to describe the correct radial extent of an electronic wave function in the bulk or on a surface by the appropriate linear combination of these two functions.

In addition to the functions which correspond to occupied atomic orbitals, so-called polarization functions are often required in order to account for the non-sphericity of the wave functions and charge density around each atom in a solid or on a surface. The concept of polarization functions is well known in quantum chemistry and also applies to solid state and surface calculations. For example, $3 d$ polarization functions are important to obtain an adequate description of the Si-Si bond length. The absence of polarization functions would cause the calculated bond length to be too long by about $0.1 \AA$ or more.

Localized basis functions such as Gaussians, STO's or numerical functions are chemically appealing. They are well suited for the description of open structures such as zeolites and the study of surfaces interacting with molecules. However, localized atomic orbitals are not efficient to describe close-packed bulk systems such as metals and alloys. For these systems one would need extended atomic orbitals with slowly decaying tails to capture the metallic delocalized character of the valence electrons. This implies that each basis function would overlap with many surrounding atoms. One would need to orthogonalize such an extended basis functions to all core functions of each of the surrounding atoms, which can be computationally demanding. In other words, localized basis functions with slowly decaying tails are not a good choice for close-packed metals whereas plane waves or augmented plane waves are a more efficient choice for this type of systems.

In the context of pseudopotential plane wave methods, the Car-Parrinello ab initio molecular dynamics method was mentioned. Fundamentally, there is no reason to use pseudopotential plane wave methods for this type of molecular dynamics. In fact, the only requirement is the possibility for rapid and numerically stable evaluation of total energies and forces. Using the concepts of the Harris functional [57], such a method has been implemented [58] using a linear combination of numerical atomic orbitals in analogy to the DMol approach. Since the total energy reaches a minimum for the exact density, any deviation of a trial density from the exact density should only have second order effects on the total energy. In the Harris functional, the total energy is written in a form which exploits this variational character of the Kohn-Sham total energy. It is then possible to perform meaningful total energy calculations by using just a superposition of atomic density without performing self-consistency calculations. If necessary, the total density can be optimized in an iterative procedure. It turns out that in many cases meaningful structures can be obtained, especially if the bonding topology is unclear and a simulated annealing or molecular dynamics calculation is required to scan the energy hyper-surface.

\section{EXAMPLES}

\subsection{Reactive adsorption of $\mathrm{SiH}_{4}$ on the $\mathrm{Si}(001)$ Surface}

The first example is related to the reaction of a silane molecule with a silicon surface. The technologically most important surface of silicon is $\mathrm{Si}(001)$. For semiconductor device applications, pure $\mathrm{Si}$ is grown by chemical vapor deposition (CVD) using molecular precursors such as dichlorosilane, $\mathrm{SiH}_{2} \mathrm{Cl}_{2}$. For the formulation of predictive kinetic models, which are needed to optimize the CVD processes, it is very useful to have a detailed and quantitative knowledge of the adsorption, dissociation, diffusion, reaction, and dissociation processes. It was the aim of the present calculations [59] to explore the capabilities of ab initio density functional calculations to provide these data.

The $\mathrm{Si}(001)$ surface is known to reconstruct in the form of a $(2 \times 1)$ structure involving a dimerization of the surface silicon atoms [60]. In the present study [59], the chemisorption of silane, $\mathrm{SiH}_{4}$, was studied 
by a first-principles density functional approach using a finite cluster model (cf. Fig. 4). The density functional equations are solved with an all-electron approach and an expansion of the one-particle wave functions in a linear combination of numerical atom orbitals as implemented in the DMol program [55,56]. For the Si atoms, $d$-polarization functions were added to the double-numerical basis set. The core electrons were treated in a frozen core approximation. The geometry optimization was carried out on the local density functional level using the explicit exchange-correlation terms given by Vosko, Wilk, and Nusair [61]. In the geometry optimization the top-most $11 \mathrm{Si}$ atoms with their hydrogen atoms are relaxed and the remaining Si atoms, which would be attached to the bulk atoms are kept at their bulk position. As can be seen from Fig. 4, the bond of the Si-dimer with the $\mathrm{SiH}_{3}$ and $\mathrm{H}$ attached opens up by about $0.3 \AA$ whereas the bond length in the adjacent Si dimer remains at the same value as on the clean surface [60]. This implies that the interaction of a silyl radical with a reconstructed $\mathrm{Si}(001) 2 \times 1$ surface is of a fairly local nature and does not lead to a breaking of dimer bonds.

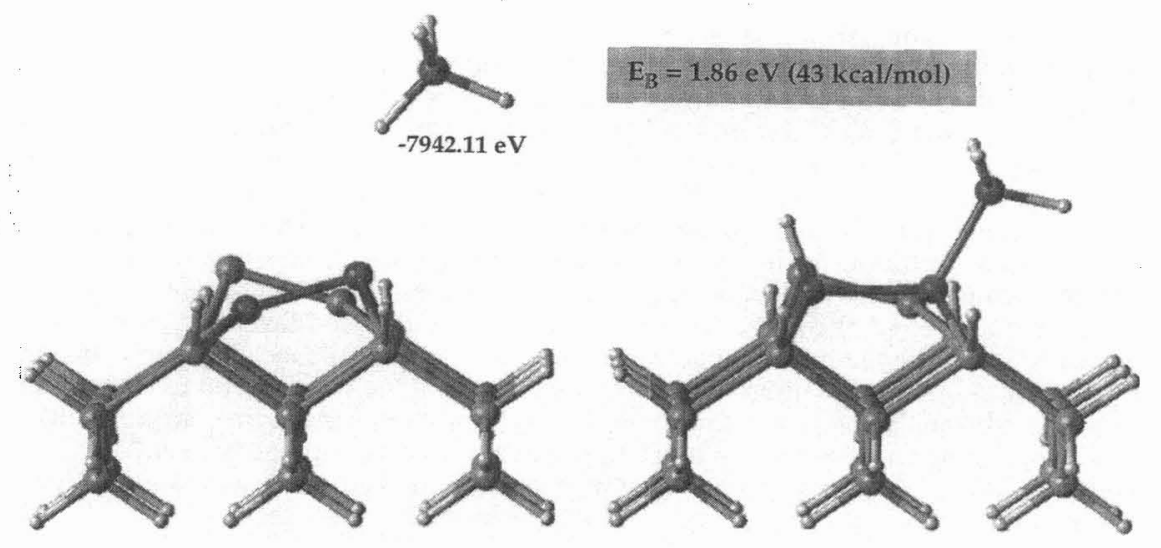

Figure 4: Dissociative adsorption of an $\mathrm{SiH}_{4}$ molecule on a reconstructed $\mathrm{Si}(001)$ surface. The surface is represented by a cluster with hydrogen atoms saturating the bonds towards the bulk. Note the antisymmetric buckled dimers on the model of the clean surface (left). Upon chemisorption of the $\mathrm{SiH}_{3}$ group on one $\mathrm{Si}$ atom of a dimer and $\mathrm{H}$ atom on the other $\mathrm{Si}$ atom of that dimer, the buckling is removed. The geometry of the neighboring Si-dimer is essentially unaffected by the chemisorption. The GGA total energies as obtained from DMol calculations are given below each system.

The chemisorption energy of a silane molecule on a $\mathrm{Si}(001) 2 \times 1$ surface is calculated by subtracting the total energy of the clean $\mathrm{Si}(001) 2 \times 1$ surface and that of an isolated silane molecule from the total energy of the cluster shown in Fig. 4 . To this end, the geometry of a cluster representing the clean surface and that of an isolated silane molecule were optimized at the same level of theory. It is known that the local density approximation leads to good geometries, but tends to overestimate dissociation energies. Therefore, the total energies of all three systems, i.e. the clean cluster, the isolated silane molecule, and the chemisorbed system are recalculated using a gradient-corrected form for the exchange-correlation potential as given by Becke [9] for the exchange and Lee, Yang, and Parr [11] for the correlation energies. From this approach, a chemisorption energy of $1.86 \mathrm{eV}$ per silane molecule is obtained.

\subsection{Reconstruction of the $\beta$-SiC(001) Surface}

Silicon carbide offers an intriguing combination of mechanical, chemical, and electronic properties. The compound is well known for its great hardness, its high thermal stability and melting point, and its chemical stability. $\mathrm{SiC}$ is a wide band gap semiconductor with important applications for high voltage and high temperature devices. Furthermore, unlike most epitaxial substrates, the lattice constant of SiC provides a good match with $\mathrm{GaN}$. However, pure single crystals of $\mathrm{SiC}$ are difficult to synthesize thus hampering their wider use as electronic material. Furthermore, SiC exists in a cubic $\beta$-form as well as a hexagonal $\alpha$ form with a large number of polytypes with significantly varying electronic properties. For these reasons, 
$\mathrm{SiC}$ and its surfaces represent a fascinating topic for experimental and theoretical studies [62]. Despite significant effort, many of the surface reconstructions of $\mathrm{SiC}$ are not known or understood.

$\mathrm{Ab}$ initio molecular dynamics and simulated annealing methods offer a unique tool to explore the possible surface reconstructions. As an illustration of this capability, we have performed a simulated annealing study of a C-terminated $\beta-\mathrm{SiC}(001)$. The surface is modeled by a nine-layer slab which is twodimensionally periodic in the form of a $(2 \times 2)$ supercell. The slab is repeated in the direction perpendicular to the surface thus forming a super-slab geometry. In the present case, the electronic structure, total energies and forces are evaluated with the Harris-functional [57] as implemented in the Fast_Structure/Sim_Ann program [58].
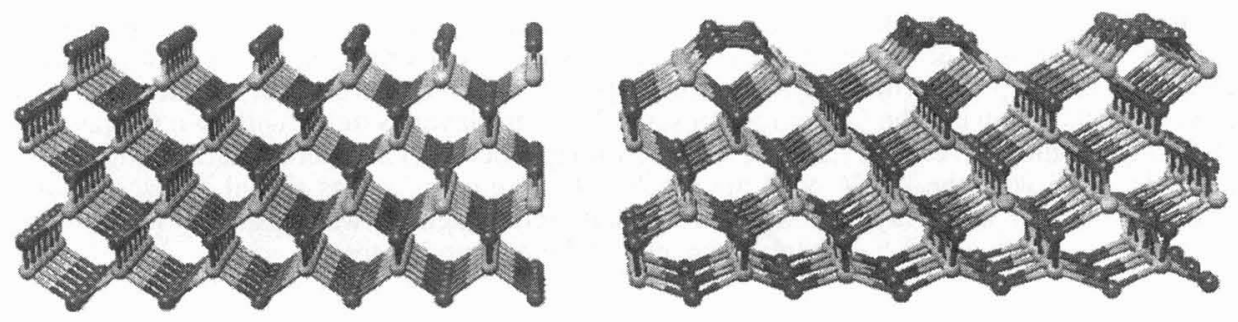

Figure 5: Determination of the reconstruction of the C-terminated $\beta$-SiC(001) surface by the Harris functional method [57] as implemented in the Fast_Structure/Sim_Ann program [58]. The left panel shows the unreconstructed surface and the right panel displays a snapshot of the simulated annealing close to the final geometry.

The simulation is started from an ideal, unreconstructed surface which is constructed by truncating the bulk crystal (cf. Fig. 5). Without any particular bias, the simulated annealing procedure leads to a dimerization of the surface $\mathrm{C}$ atoms thereby creating a $\mathrm{c}(2 \times 1)$ structure (cf. Fig. 5). In this calculation, only superposed spherical atomic densities are used to construct the effective potential. Given the mixed covalent and ionic character of $\mathrm{SiC}$, this is a rather crude approximation. It is surprising and reassuring to see that the major features of the bonding mechanism are already captured by this model, while the details of the geometric parameters deviate from self-consistent calculations. For example, the $\mathrm{C}-\mathrm{C}$ bond distance is found to be about $1.20 \AA$ compared with the value of $1.36 \AA$ reported for accurate self-consistent pseudopotential calculations using Gaussian-type basis sets [47]. The Si-C back-bond between the surface and sub-surface atoms is obtained to be $1.94 \AA$ compared with $1.86 \AA$ from the self-consistent calculations. In the interior of the slab, the Harris functional gives a Si-C distance of $1.84 \AA$ compared with the experimental value of $1.883 \AA$. As one can see, the non-self-consistent Harris functional approach gives the correct topology and deviations between $0.04-0.10 \AA$ in bond distances compared with fully selfconsistent density functional calculations. This demonstrates that simplified, but parameter-free quantum mechanical approaches based on the Harris functional can be used to identify the topology of low-energy structures. Further geometry optimizations using more accurate (and more time consuming) methods can subsequently be applied to refine the details of a structure and to obtain reliable energies.

\subsection{Magnetism on Surfaces and Interfaces}

The third example is taken from the area of magnetism. Ab initio density functional theory has achieved major successes in the study of magnetism on surfaces and interfaces. In fact, in the 1970's there were experimental claims of "magnetically dead" layers on the surfaces of $\mathrm{Ni}$ and $\mathrm{Fe}$. As accurate electronic structure calculations on transition metal surfaces were developed in the late 1970's and applied to this question, it became obvious that the reduced dimensionality near a $\mathrm{Ni}$ or Fe surface must lead to an enhancement of the local magnetic moments rather than to dead layers. Accurate approaches such as the FLAPW method clearly gave enhanced magnetic moments thereby contradicting the earlier experiments [63]. It became evident that the earlier experiments did not have clean, but contaminated metal surfaces. As soon as atomically clean surfaces became experimentally accessible, the agreement between the experimental data and the theoretical predictions was re-established.

The accuracy of the FLAPW method allows the calculation of extremely subtle magnetic effects such as the magnetocrystalline anisotropy (MCA) energy [64]. In these calculations, the spin-orbit coupling is included in the form of a perturbational treatment and a state-tracking method is applied to reduce the large 
number of k-points which would be needed if a "blind" Fermi filling would be used. It was found [64] that for an $\mathrm{Fe}(001)$ monolayer, the angular dependence of the MCA varies only by $0.46 \mathrm{meV}$ as the angle between the magnetic moment and the surface normal is changed from 0 to $90^{\circ}$.

Recording technology is one of the major driving forces for the search for novel magnetic materials. Transition metal surfaces, atomic overlayers, sandwich structures, and artificially layered materials present particularly fascinating possibilities since the magnetic properties can be tailored through the control of the epitaxial process. As illustrated above, first principles local spin density functional calculations have been highly successful in the prediction of the magnetic properties of these systems with reduced dimensionality. The interest in magneto-optical recording and the discovery of the giant magnetoresistance effect have intensified the interest in these systems and their investigation by electronic structure calculations [65].

An example of the present computational capabilities is the magnetic ordering in $\mathrm{Co} / \mathrm{Cu}$ multilayers [66]. Depending on the thickness of the non-magnetic $\mathrm{Cu}$ layers, the Co layers couple either ferromagnetically or anti-ferromagnetically. In the hexagonal Co structure, the nearest-neighbor Co-Co distance is $2.51 \AA$ compared with $2.54 \AA$ for fcc copper. In the calculations [66], an fcc superlattice of Co and $\mathrm{Cu}$ was created by using the $\mathrm{Cu}$ lattice constant. For multilayer systems with two Co layers and 2,3 , 4 , and $5 \mathrm{Cu}$ layers, the total energy difference for ferromagnetic $(\mathrm{FM})$ and antiferromagnetic $(\mathrm{AF})$ coupling has been calculated with the ASW-ASA approach [37]. The calculations reveal an oscillation with the systems containing 2,3 and 6 layers being preferentially ferromagnetic whereas the systems with 3 and 4layer $\mathrm{Cu}$ layers are predicted to be antiferromagnetic in their ground states.

\section{SUMMARY, CONCLUSIONS, AND OUTLOOK}

Driven by the technological need to control surfaces and interfaces on the atomic scale, theoretical and computational tools are becoming increasingly important in order to interpret experiments and to make predictions prior to the actual synthesis of a system. To this end, density functional theory has emerged as a powerful tool to predict not only the electronic structure, but perhaps even more importantly, to determine the geometries of surfaces, adsorbates, and interfaces and to provide thermochemical data such as binding energies and diffusion barriers, which are difficult to get from experiment.

With current ab initio density functional methods it is possible to predict interatomic distances which agree within a few hundreds of one Ångström with experiment. This is true for bulk solids, surfaces, interfaces, and molecules. At present, systems with up to about 100 atoms can be treated on this level of theory. Using so-called gradient corrected density functionals, binding energies can be predicted with an accuracy of about $5 \mathrm{kcal} / \mathrm{mol}$. Relative energy differences can be obtained at an even higher level of accuracy. This capability was illustrated here by the dissociative adsorption of a silane molecule on a $\mathrm{Si}(001)$ surface. The calculations show that the chemical interactions seem to be fairly localized, since the geometry of the Si-dimer next to the one involved in a Si-Si bond formation remains almost unchanged. It can be anticipated that this type of calculations will be very useful in developing better thermodynamic and kinetic models for the simulation of processes such as chemical vapor deposition, sorption, catalysis, and corrosion.

The ability to predict surface structures was illustrated here by the $c(2 \times 1)$ reconstruction of the $\beta$ $\mathrm{SiC}(001)$ surface. By using the Harris functional, which is a simplified density functional approach, it was found that a simulated annealing procedure starting with atomic positions of the truncated bulk crystal, the $\mathrm{C}$-rich surface spontaneously forms $\mathrm{C}-\mathrm{C}$ dimers thus forming a $\mathrm{c}(2 \times 1)$ pattern. The Harris functional method thus gives correctly the topology of this low-energy structure. Further refinement of the structure is then possible with self-consistent density functional methods such as the pseudopotential plane wave approach. The combination of the approximate Harris functional method together with self-consistent approaches represent a powerful combination which allows the exploration of novel systems where little structural information is available.

The third group of examples is taken from surface and interface magnetism. At present, methods such as the FLAPW approach allow the accurate calculation of magnetic moments, spin densities, and spinpolarized energy band structures. Even the prediction of subtle effects such as the crystalline magnetic anisotropy is possible with this level of theory.

In general, $a b$ initio density functional theory has now matured to the point where reliable structural, electronic, and magnetic properties can be obtained for a large number of systems including metals, semiconductors, oxides and other inorganic compounds, but also purely organic systems such as molecules and molecular crystals. However, a number of obstacles remain to be overcome in order to link atomistic simulations with the macroscopic world. The size of systems which can be treated quantum mechanically is still rather small, namely of the order of hundred atoms. Substantial efforts are currently under way to 
develop so-called order- $N$ methods for which the computational effort scales linearly with the number of atoms. However, as one increases the number of atoms, for example to describe the interface between and amorphous polymer and a metal oxide surface, it is obvious that the problem is not only the calculation of a large system, but rather the exploration of the entire conformational space with all its degrees of freedom. In fact, the description of time-dependent phenomena such as diffusion and chemical reactions becomes the principle challenge. Even if one would increase the computational speed of ab initio methods by three orders of magnitudes - which will probably happen within the coming decade - the problem of bridging the gap between the femto-seconds of atomistic processes and hours and years of macroscopic processes will not be solved alone by faster computers and algorithms. Novel theoretical concepts are called for.

Another challenge is the accuracy which can be achieved with current many-electron approaches. The most accurate quantum mechanical methods such as coupled-cluster theory still has errors of the order of 1 $\mathrm{kcal} / \mathrm{mol}$ which is very accurate when compared with the total energy of a system, but still large when applied to the determination of chemical reaction rates. Within density functional theory, the generalized gradient corrections have provided a significant progress, but they are not yet good enough for describing reaction barriers, for example in proton transfer reactions. Another qualitative step similar to the one from the LDA to the GGA is called for. Possibly, density functionals can be created with input from accurate quantum chemical or quantum Monte Carlo methods.

Ultimately, progress in theoretical and computational techniques is coupled with the advances in experimental techniques. Given the impressive evolution of surface techniques including real space probes, the continuing dialog between theorists and experimentalists will ensure the progress in the exciting and important field of surfaces and interfaces of advances materials.

\section{Acknowledgments}

It is a great pleasure to acknowledge the help and fruitful discussions with many colleagues and friends, especially Bernard Delley, Ludovic Douillard, Arthur J. Freeman, Catalina Guerra, John Harris, Patrick Soukiassian, and Jürgen Sticht.

\section{References}

[1] Wimmer, E., J. Comp.-Aided Mat. Design 1 (1993) 215.

[2] Wimmer, E., Materials Science and Engineering B37 (1996) 72.

[3] Parr, R. G.; and Yang, W.Density-Functional Theory of Atoms and Molecules (Oxford University Press, New York, NY, 1989)

[4] Hohenberg, P. and Kohn, W., Phys. Rev. 136 (1964) B864.

[5] Kohn, W. and Sham, L. J., Phys. Rev. 140 (1965) A1133.

[6] Kohn, W., Phys. Rev. Lett. 76 (1996) 3168.

[7] Ceperley, D. M., and Alder, B. J., Phys. Rev. Lett. 45 (1980) 566.

[8] Becke, A., J. Chem. Phys. 84 (1986) 4524.

[9] Becke, A., Phys. Rev. A 38 (1988) 3098.

[10] Perdew, J. P., Phys. Rev. B 33 (1986) 8822.

[11] Lee, C., Yang, W., and Parr, R. G., Phys. Rev. B 37 (1988) 786.

[12] von Barth, J., and Hedin, L. J. Phys. C 5 (1972) 1629.

[13] Gunnarsson, O., Lundqvist, B.I., and Lundqvist, S., Solid State Commun. 11 (1972) 149.

[14] Hybertsen, M. S. and Louie, S. G., Phys. Rev. Lett. 58 (1987) 1551 and references therein.

[15] Anisimov, V. I., Zaanen, J., and Andersen, O. K., Phys. Rev. B 44 (1991) 943.

[16] Zunger, A., Phys. Rev. B 22 (1979) 649; Svane, A., Phys. Rev. Lett. 72 (1994) 1248.

[17] Runge, E., and Gross, E. K. U., Phys. Rev., Lett. 52 (1984) 997; Wacker, O. J., Kümmel, R., and Gross, E. K. U., Phys. Rev. Lett. 73 (1994) 2915.

[18] Koelling, D. D., and Harmon, B. N., J. Phys. C 10 (1977) 3107.

[19] Car, R. and Parrinello, M., Phys. Rev. Lett. 55 (1985) 2471.

[20] Payne, M. C., Teter, M. P., Allan, D.C., and Joannopoulos; J. D., Rev. Mod. Phys. 64 (1992) 1045.

[21] Furthmüller, J., Kresse, G., Hafner, J., Stumpf, R., and M. Scheffler, M., Phys. Rev. Lett. 74 (1995) 5084.

[22] Stampfl, C., and Scheffler, M., Phys. Rev. B 54 (1996) 2868.

[23] Slater, J. C. Phys. Rev. 51 (1937) 846.

[24] Koelling, D. D., and Arbman, G. O., J. Phys. F 5 (1975) 2041. 
[25] Andersen, O. K. (1975) Phys. Rev. B 12, 3060.

[26] Krakauer, H., Posternak, M., and Freeman, A.J., Phys. Rev. B 19 (1979) 1706.

[27] Weinert, M., J. Math. Phys. 22 (1981) 2433; Wimmer, E., Krakauer, H. Weinert, M. and Freeman, A.J., Phys. Rev. B 24 (1981) 864.

[28] Weinert, M., Wimmer, E., and Freeman, A. J., Phys. Rev. B 26 (1982) 4571.

[29] Soler, J. M., Williams, A. R., Phys. Rev. B 43 (1991) 6411.

[30] Yu, M., Krakauer, H., and Singh, D., Phys. Rev. B 45 (1992) 8671.

[31] Blaha, P., Schwarz., K., Sorantin, P., and Trickey, S. B. Comput. Phys. Commun. 59 (1990) 399.

[32] Wang, D.-S., Freeman, A. J., and Krakauer, H., Phys. Rev. B 26 (1982) 1340.

[33] Wu, R., and Freeman, A. J., Phys. Rev. Lett. 73 (1994) 1994.

[34] Korringa, J., Physica 13 (1947) 392.

[35] Kohn, W., and Rostoker, N., Phys. Rev. 94 (1954) 1111.

[36] Skriver, H. L., The LMTO method (Springer Verlag, 1984).

[37] Williams, A. R., Kübler, K., and Gelatt, J. R.; Phys. Rev. B 19 (1979) 6094.

[38] Methfessel, M., Phys. Rev. B 38 (1988) 1537.

[39] Eyert V., Thesis, (Technische Hochschule Darmstadt, German, 1991).

[40] Dovesi, R., Saunders, V.R., and Roetti, C., CRYSTAL 92, User Manual, (Univ, of Turin, Italy, and SERC Daresbury Laboratory, U.K., 1992).

[41] Wang, C. S., and Callaway, J., Phys. Rev. B 8(1974) 4897.

[42] Feibelman, P. J., Phys. Rev. Lett. 54 (1985) 2627.

[43] Sambe, H., and Felton, R. H., J. Chem. Phys. 62 (1975) 1122.

[44] Dunlap, B.I., J. Phys. Chem. 90 (1986) 5524.

[45] Salahub, D. R. in Ab Initio Methods in Quantum Chemistry-II, edited by Lawley, K. P., J. (Wiley \& Sons, New York, 1987) p. 447.

[46] Andzelm, J., and Wimmer, E., J. Chem. Phys. 96 (1992) 1280.

[47] Sabisch, M., Krüger, P., Mazur, A., Rohlfing, M., and Pollmann J., Phys. Rev, B 53 (1996) 13121.

[48] Hay, P. J., Wadt, W. R., and Dunning, T. H., Annu. Rev. Phys. Chem. 30 (1979) 4311.

[49] Godbout, N., Salahub, D. R., Andzelm, J., and Wimmer, E., Can. J. Chem. 70 (1992) 560.

[50] Baerends, E.J., Ellis, D. E., and Ros, P. (1973) Chem. Phys. 2, 41.

[51] Rosen, A., and Ellis, D. E. J. Chem. Phys. 65 (1976) 3629.

[52] te Velde, G., and Baerends, E. J., Phys. Rev. B 44 (1991) 7888.

[53] Averill, F. W., and Ellis, D. E., J. Chem. Phys. 59 (1973) 6412.

[54] Delley, B., and Ellis, D. E., J. Chem. Phys. 76 (1982) 1949.

[55] Delley, B., J. Chem. Phys. 92 (1990) 508.

[56] Delley, B., J. Chem. Phys. 94 (1991) 7245.

[57] Harris, J., Phys. Rev. B 31 (1985) 1770.

[58] Fast_Structure User's Guide, (MSI, San Diego, 1996)

[59] Guerra C., and Wimmer, E., unpublished

[60] Tang, S., Freeman, A. J., and Delley, B., Phys. Rev. B 45 (1992) 1776 and references therein.

[61] Vosko, S, H., Wilk, L., and Nusair, M. Can. J. Phys. 58 (1980) 1200.

[62] Semond, F., Soukiassian, P., Mayne, A., Dujardin, G., Douillard, L., and Jaussaud, C., Phys. Rev. Lett. 77 (1996) 2013 and references therein.

[63] Jepsen, O., Madsen, M., and Andersen, O.K., J. Magn. Magn. Mater. 15-18 (1980), 867; Krakauer, H., Freeman, A. J., and Wimmer, E., Phys. Rev. B 28 (1983), 610; Wimmer, E., Freeman, A. J., and Krakauer, H., Phys. Rev. B, 30 (1984), 3113 and references therein.

[64] Wang, D.-S., Wu, R., and Freeman, A. J., Phys. Rev. Lett. 70 (1993) 869.

[65] Jansen, H. J. F., Physics Today 48 (1995) 50.

[66] Herman, F., Van Schilfgarde, M., and Sticht, in Physics of Transition Metals vol 1 and 2 (World Scientific, 1993) p. 425, J., Oppeneer, P., and Kübler, J., editors, reprinted from the Int. J. of Modern Physics B7 (1993). 